\title{
In silico screening of drug-membrane thermodynamics reveals linear relations between bulk partitioning and the potential of mean force
}

\author{
Roberto Menichetti, ${ }^{1,}$ a) Kiran H. Kanekal, ${ }^{1}$ Kurt Kremer, ${ }^{1}$ and Tristan Bereau ${ }^{1,}$ b) \\ Max Planck Institute for Polymer Research, Ackermannweg 10, 55128 Mainz, \\ Germany
}

(Dated: 28 July 2021)

The partitioning of small molecules in cell membranes - a key parameter for pharmaceutical applicationstypically relies on experimentally-available bulk partitioning coefficients. Computer simulations provide a structural resolution of the insertion thermodynamics via the potential of mean force, but require significant sampling at the atomistic level. Here, we introduce high-throughput coarse-grained molecular dynamics simulations to screen thermodynamic properties. This application of physics-based models in a large-scale study of small molecules establishes linear relationships between partitioning coefficients and key features of the potential of mean force. This allows us to predict the structure of the insertion from bulk experimental measurements for more than 400,000 compounds. The potential of mean force hereby becomes an easily accessible quantity - already recognized for its high predictability of certain properties, e.g., passive permeation. Further, we demonstrate how coarse graining helps reduce the size of chemical space, enabling a hierarchical approach to screening small molecules.

\section{INTRODUCTION}

The thermodynamic partitioning of small molecules in lipid-bilayer membrane systems is a key parameter for assessing their suitability for pharmaceutical applications ${ }^{1] 3}$ It follows that the thermodynamic partitioning is extremely relevant for high-throughput screening approaches to drug discovery. Experimentally, for different drug molecules it is typically obtained by measuring the bulk concentrations of the molecules in various systems that act as a proxy for the aqueous lipid membrane systems (e.g., oil/water or Caco-2 cell assays)! !14 The same property is obtained in silico by simulating the small molecule in an aqueous environment in the vicinity of a lipid-bilayer membrane (Fig.1p). Further, structural resolution is provided by the potential of mean force, which describes how the free energy of the system changes as a function of certain reaction coordinates (Fig. 1 k). In the context of drug-membrane interactions, the reaction coordinate is often the position of the molecule normal to the membrane ${ }^{112}$ Several studies have investigated drugmembrane interactions using atomistic molecular dynamics simulations of specific sets of biomolecules (e.g., amino acids or drug molecules) ${ }^{5}+8$ Moreover, by means of an integrated measure, atomistic potentials of mean force were shown to accurately reproduce the experimental transfer free energies between water and membrane for different compounds $[9$

However, these studies have proven to be severely computationally expensive, with roughly $10^{5} \mathrm{CPU}$ hours needed to estimate the potential of mean force for each compound, even with the use of enhanced sampling techniques (e.g., umbrella sampling) $\stackrel{6110}{{ }^{6}}$ Given the extensive computational resources required for these systems,

\footnotetext{
a) Electronic mail: menichetti@mpip-mainz.mpg.de

b) Electronic mail: bereau@mpip-mainz.mpg.de
}

a high-throughput scheme based on atomistic molecular dynamics simulations is currently unfeasible for spanning the large regions of chemical compound space needed to obtain broadly applicable structure-property relationships.

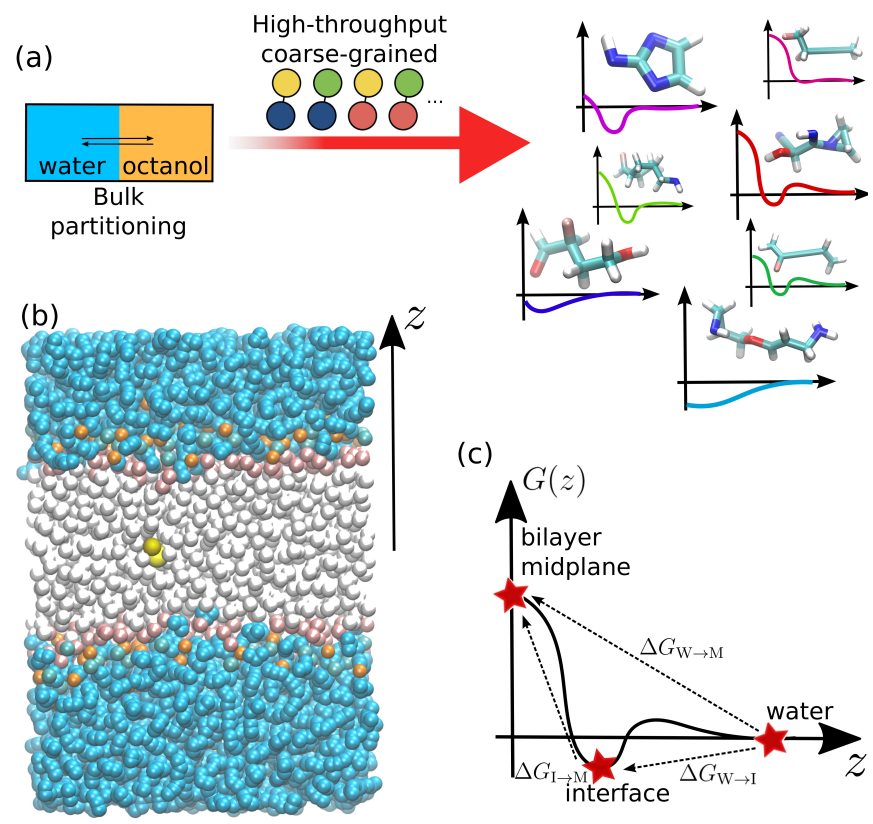

FIG. 1. (a) This work establishes linear relationships allowing to predict key features of the potential of mean force from a compound's bulk water/octanol partitioning coefficient, identified from high-throughput coarse-grained simulations. (b) Simulation setup of a small molecule (yellow) partitioning between water (light blue) and the lipid membrane (white). (c) Potential of mean force and the three thermodynamic environments of interest: the bilayer midplane, the membrane-water interface, and the water phase.

Coarse-grained molecular dynamics simulations pro- 
vide a means to significantly reduce the computational expense of fully atomistic simulations while still capturing the relevant physical properties $\frac{11112}{11}$ Coarse-grained representations of molecules result from mapping groups of atoms to coarse-grained "pseudo-atoms" or beads. The interaction potentials of these beads are determined such that the essential properties of the fully atomistic system are retained at the coarse-grained level. This usually corresponds to a smoothing of the underlying atomistic free-energy landscape that preserves its relevant features, allowing for more efficient sampling. Adapting coarse-grained molecular dynamics simulations to highthroughput screening of chemical compounds requires flexible and reliable mapping and force-field parameterization methods. The coarse-grained Martini force field provides a robust set of transferable force-field parameters for a variety of biomolecular systems 13114 Coarsegrained molecules - akin to a combination of functional groups - are constructed from a small set of bead types that encompass a representative spectrum of partitioning free energies between polar and apolar phases. We explore a small subset of chemical-compound space within this model by constructing all possible combinations of one- and two-bead molecules out of neutral bead types119 in total. We then evaluate the partitioning of each compound in an aqueous lipid-bilayer system from potentials of mean force and free-energy differences. Our computational high-throughput screening scheme establishes linear relationships between key features of the potential of mean force across compounds, allowing a semi-quantitative estimation given the Martini representation of the compound and a single parameter: the water/octanol partitioning coefficient-an experimentallyaccessible bulk property. Further, the 119 coarse-grained molecules backmap to at least 465,387 different organic small molecules ranging from 30 to $160 \mathrm{Da}$, demonstrating a significant reduction in chemical compound space due to the limited set of bead types of the coarse-grained model. We thus present two complementary ways to estimate key features of the potential of mean force for a large number of small molecules: from coarse-grained simulations and from linear relationships established in this work.

\section{METHODS}

\section{A. Molecular dynamics simulations}

Molecular Dynamics simulations in this work were performed in GROMACS 4.6.6, ${ }^{[5]}$ using the Martini force field $[16-19$ We relied on the standard force field parameters ${ }^{20}$ with an integration time step of $\delta t=0.02 \tau$, where $\tau$ is the model's natural unit of time.

A Parrinello-Rahman barostat ${ }^{21}$ and a stochastic velocity-rescaling thermostat ${ }^{22}$ provided control over the system pressure $(P=1 \mathrm{bar})$ and temperature $(T=$ $300 \mathrm{~K})$. The corresponding coupling constants were $\tau_{\mathrm{P}}=$
$12 \tau$ and $\tau_{\mathrm{T}}=\tau$.

Bulk simulations consisted of $N_{W}=450$ and $N_{O}=336$ water and octane molecules, respectively. A DOPC membrane of $36 \mathrm{~nm}^{2}$ was generated by means of the INSANE building too $\frac{23}{23}$ and subsequently minimized, heated up, and equilibrated. The total number of lipids in the membrane was $N_{L}=128$ (64 per layer), immersed in $N_{W}^{\prime}=1590$ water molecules. As usual when using nonpolarizable Martini water, we added an additional $10 \%$ of antifreeze particles in the simulations containing water molecules ${ }^{17}$

In the case of two-bead molecules, we first considered a representative subset of 40 coarse-grained compounds, roughly uniformly covering a range of transfer freeenergies from water to bilayer midplane of $\Delta G_{\mathrm{W} \rightarrow \mathrm{M}} \simeq$ $[-8,14] \mathrm{kcal} / \mathrm{mol}$. We determined the corresponding potentials of mean force as a function of the distance $z$ of the compound from the bilayer midplane, $G(z)$, by means of umbrella-sampling techniques. ${ }^{24}$ We set biasing potentials with a harmonic constant of $k=240$ $\mathrm{kcal} / \mathrm{mol} / \mathrm{nm}^{2}$ every $0.1 \mathrm{~nm}$ along the normal to the bilayer midplane, for a total of 24 simulations. In each of them, two solute molecules were placed in the membrane in order to increase sampling and alleviate leaflet area asymmetry. ${ }^{[9 \mid 25]}$ The total production time for each umbrella simulation was $1.2 \cdot 10^{5} \tau$. We estimated the free-energy profiles by means of the weighted histogram analysis method, 26 28 and the corresponding errors via bootstrapping. ${ }^{29}$ The same calculations were performed in order to determine the potentials of mean force for all of the 14 single-bead compounds analyzed in this work. The computational cost for the reconstruction of each potential of mean force amounted roughly to $200 \mathrm{CPU}$ hours.

In the comparison with atomistic resultt5 ${ }^{5}$ presented in Fig. 3 and Fig. 5 , we horizontally shifted the coarsegrained potentials of mean force by up to $0.4 \mathrm{~nm}$ to correct for discrepancies in the bilayer thickness between the atomistic and the coarse-grained model, see Ref. 30.

We herein focus on calculating $\Delta G_{\mathrm{W} \rightarrow \mathrm{I}}$ and $\Delta G_{\mathrm{I} \rightarrow \mathrm{M}}$, the transfer free-energies between the three different environments - water (W), interface (I), and bilayer midplane $(\mathrm{M})$ - along the potential of mean force, see Fig. 1. In terms of $G(z)$, these are defined as $\Delta G_{\mathrm{W} \rightarrow \mathrm{I}}=G(\bar{z})-$ $G(z \rightarrow \infty)$ and $\Delta G_{\mathrm{I} \rightarrow \mathrm{M}}=G(z=0)-G(\bar{z})$, where $\bar{z} \approx 1.8 \mathrm{~nm}$ is the position of the lipid-water interface with respect to the bilayer midplane $(z=0)$.

The transfer free energies for all 105 coarse-grained two-bead molecules were determined from alchemical transformations. ${ }^{31}$ Given the excellent agreement between the two end points of a potential of mean force and the water/octane partitioning (see Sec. III A and Fig. 2), as already pointed out in Ref. 32, the latter was used as a proxy for the hydrophobic core of the membrane.

For two compounds $A$ and $B$, their respective water/interface and interface/octane transfer free energies are denoted by $\Delta G_{\mathrm{W} \rightarrow \mathrm{I}}^{A}, \Delta G_{\mathrm{W} \rightarrow \mathrm{I}}^{B}, \Delta G_{\mathrm{I} \rightarrow \mathrm{O}}^{A}$ and $\Delta G_{\mathrm{I} \rightarrow \mathrm{O}}^{B}$. We can relate these quantities to alchemical transforma- 
tions from $A$ to $B$ in fixed environments via

$$
\begin{gathered}
\Delta G_{\mathrm{W} \rightarrow \mathrm{I}}^{B}=\Delta G_{\mathrm{W} \rightarrow \mathrm{I}}^{A}+\left(\Delta G_{\mathrm{I}}^{A \rightarrow B}-\Delta G_{\mathrm{W}}^{A \rightarrow B}\right), \\
\Delta G_{\mathrm{I} \rightarrow \mathrm{O}}^{B}=\Delta G_{\mathrm{I} \rightarrow \mathrm{O}}^{A}+\left(\Delta G_{\mathrm{O}}^{A \rightarrow B}-\Delta G_{\mathrm{I}}^{A \rightarrow B}\right) .
\end{gathered}
$$

We computed $\Delta G_{\mathrm{I}}^{A \rightarrow B}, \Delta G_{\mathrm{W}}^{A \rightarrow B}$ and $\Delta G_{O}^{A \rightarrow B}$ by means of separate molecular dynamics simulations at the interface, in bulk water and in bulk octane. By performing a linear sequence of such transformations covering all 105 two-bead compounds, we were able to determine the full set of $\Delta G_{\mathrm{W} \rightarrow \mathrm{I}}$ and $\Delta G_{\mathrm{O} \rightarrow \mathrm{I}}$ analyzed in this work.

In the calculation of the $\Delta G_{j}^{A \rightarrow B}, j=\mathrm{I}, \mathrm{W}, \mathrm{O}$, we employed the multistate Bennett acceptance ratid 33 (MBAR), a generalization of the BAR method ${ }^{34}$ MBAR determines the free energy difference $\Delta G_{j}^{A \rightarrow B}$ by appropriately combining the results obtained from simulations that sample the statistical ensembles generated by a set of interpolating Hamiltonians $H(\lambda), \lambda \in[0,1]$, with $H(\lambda=0)=H^{A}$ and $H(\lambda=1)=H^{B}$. Specifically, we made use of 21 evenly distributed $\lambda$-points between 0 and 1 for each alchemical transformation and in each environment (interface, water and octane). The production time for each $\lambda$ point was $2 \cdot 10^{4} \tau$ in bulk water and bulk octane and $4 \cdot 10^{4} \tau$ at the interface. The cumulative computational cost of performing each alchemical transformation in water, interface and octane amounted roughly to $60 \mathrm{CPU}$ hours.

Given the spatial localization of the interface, the free energy $\Delta G_{\mathrm{I}}^{A \rightarrow B}$ was computed by adding a harmonic potential between the compound and the bilayer midplane at a distance $\bar{z}=1.8 \mathrm{~nm}$.

\section{B. Analysis of chemical compound space}

The algorithm developed by Bereau and Kremer ${ }^{30}$ for the automated parameterization of the Martini force field was used to coarse grain approximately 3.5 million small organic compounds, for which 465,387 were mapped to Martini molecules consisting of one and two beads. The list of compounds was obtained from the Generated Database (GDB) of molecules which had up to 10 heavy (excluding Hydrogen) atoms,,$\sqrt[3556]{36}$ representing most synthetically-feasible small organic molecules between 30 and 160 Da. The algorithm utilizes a mapping energy function that is minimized for each molecule so as to optimize both the number and placement of beads used in its coarse-grained representation. The bead typing occurs by assigning an existing Martini bead type that has the best matched water/octanol partition coefficient with that of the molecular fragment encapsulated by the bead. The partition coefficients of these fragments are obtained by using ALOGPS $\stackrel{37}{3}$ a neural-network algorithm that predicts these values given the chemical structure of the fragment. The standard mean error associated with this algorithm is $0.36 \mathrm{kcal} / \mathrm{mol}{ }^{37 / 38}$

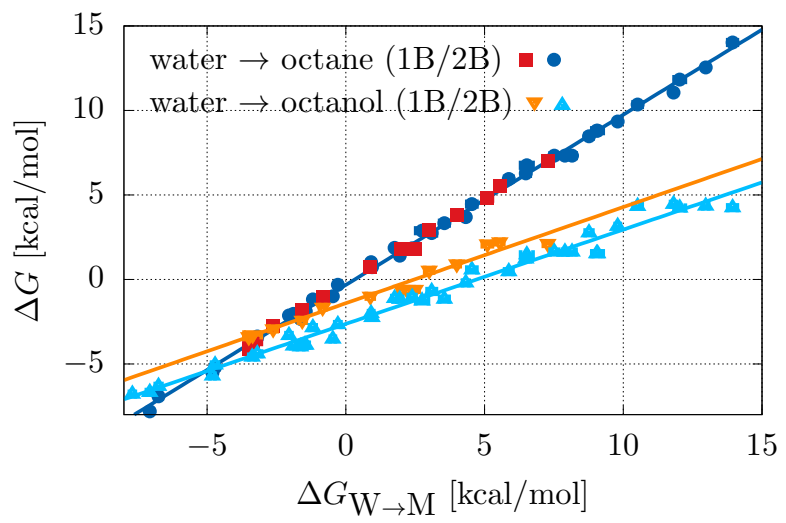

FIG. 2. Relationship between the two endpoints of the potential of mean force (i.e., $\Delta G_{\mathrm{W} \rightarrow \mathrm{M}}=G(z=0)-G(z \rightarrow \infty)$ ) with the water/octane and water/octanol partitioning free energies, in the case of one- (1B) and two-bead (2B) compounds.

\section{RESULTS}

\section{A. Linear relationships}

Fig. 2 shows the excellent agreement between the two end-points of a potential of mean force (i.e., $\Delta G_{\mathrm{W} \rightarrow \mathrm{M}}=$ $G(z=0)-G(z \rightarrow \infty))$ and the water/octane partitioning, $\Delta G_{\mathrm{W} \rightarrow \mathrm{O}}$, which illustrates that bulk octane is a good proxy to represent the hydrophobic core of the bilayer, as already discussed in Ref 32, A linear fit for the two quantities provided

$$
\Delta G_{\mathrm{W} \rightarrow \mathrm{O}}=\Delta G_{\mathrm{W} \rightarrow \mathrm{M}}-\alpha,
$$

$\alpha \approx 0.28,0.32 \mathrm{kcal} / \mathrm{mol}$ for one-bead and two-bead compounds respectively, with Pearson correlation coefficients $R^{2}=0.99$. As described in the Methods section, this allowed us to determine transfer free energies with respect to an octane environment, later converting them to the corresponding membrane values.

For every compound, the transfer free energies depicted in Fig. 11 are subject to a thermodynamic cycle that links the three variables

$$
\Delta G_{\mathrm{W} \rightarrow \mathrm{I}}+\Delta G_{\mathrm{I} \rightarrow \mathrm{M}}-\Delta G_{\mathrm{W} \rightarrow \mathrm{M}}=0 .
$$

Fig. 3 illustrates the relationship between these three transfer free energies for all 119 coarse-grained molecules considered in this work inserted in a DOPC membrane. In both cases of one- and two-bead compounds, beyond the thermodynamic cycle linking $\Delta G_{\mathrm{W} \rightarrow \mathrm{I}}, \Delta G_{\mathrm{I} \rightarrow \mathrm{M}}$ and $\Delta G_{\mathrm{W} \rightarrow \mathrm{M}}$, we observe a collapse of the data onto two lines, indicative of a linear relationship between these transfer free energies. Moreover, the only difference between one- and two-bead results consists in the presence of a simple offset (i.e., same slope) between the profiles.

As a result, the thermodynamic cycle shown in Fig. 1. 
can be reconstructed from the knowledge of a single variable and the Martini bead representation of the compound. The error in doing so amounts to $\approx 0.4 \mathrm{kcal} / \mathrm{mol}$. These relationships are validated from reference atomistic simulations of amino-acid side chains,, , where we consider only atomistic compounds whose Martini representation consists of a single bead. While most points fall within the linear fit from the single-bead coarse-grained data, we observe three statistically significant outliers: asparagine (asn), isoleucine (ile), and glutamine (gln). These molecules lie on the data corresponding to twobead compounds, although their Martini representation consists of a single bead! ${ }^{18}$ The origin of such discrepancies will be explained below. The comparison of atomistic and Martini potential of mean force for protein sidechains was already performed in Ref.18.
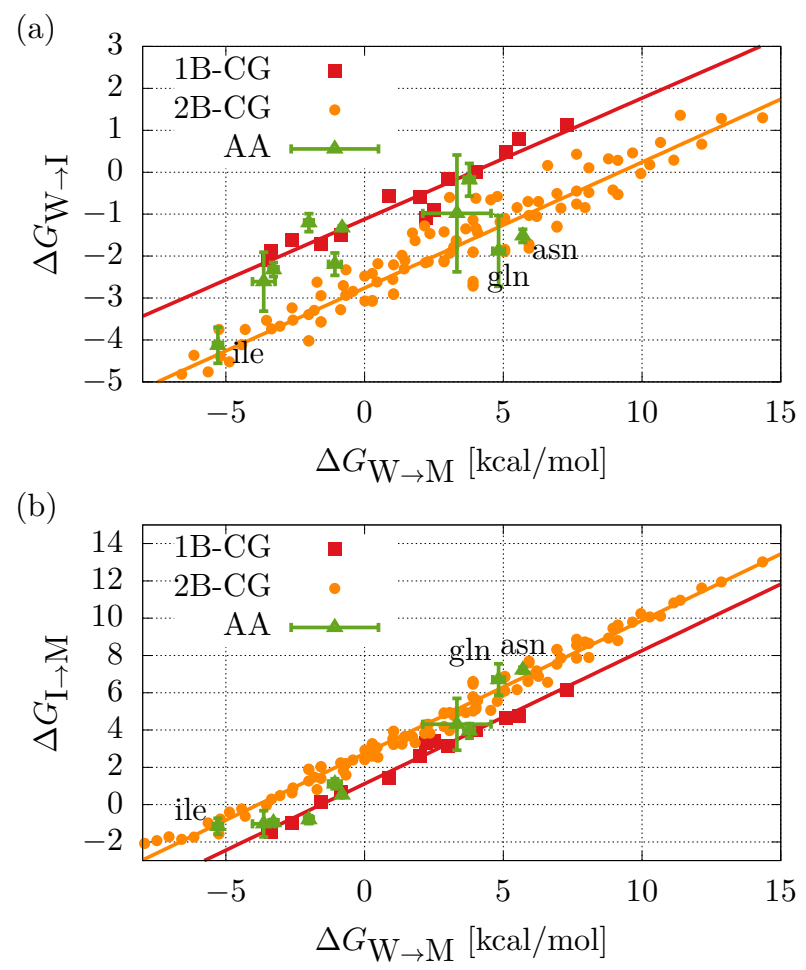

FIG. 3. (a) Transfer free energies from water to interface $\Delta G_{\mathrm{W} \rightarrow \mathrm{I}}$ as a function of the compound's water/membrane partitioning free energy, $\Delta G_{\mathrm{W} \rightarrow \mathrm{M}}$. The red and orange curve correspond to coarse-grained estimates for one-bead (1B) and two-bead (2B) molecules, respectively. The green points (AA) depict corresponding atomistic references of aminoacid side chains. ${ }^{[5}$ (b) Transfer free energies from interface to the membrane $\Delta G_{\mathrm{I} \rightarrow \mathrm{M}}$ as a function of the compound's water/membrane partitioning free energy, $\Delta G_{\mathrm{W} \rightarrow \mathrm{M}}$. Color coding follows from (a). In both figures, statistically significant outliers (see text) are marked with a label (asn, ile, and $\operatorname{gln})$.

Remarkably, the relationships between transfer free energies displayed in Fig. 3 can further be linked to a compound's water/octanol free-energy $\Delta G_{\mathrm{W} \rightarrow \mathrm{Ol}}$, given its accurate linear relation with $\Delta G_{\mathrm{W} \rightarrow \mathrm{M}}$, see Fig. 2, A fit of the data provided

$$
\Delta G_{\mathrm{W} \rightarrow \mathrm{M}}=\gamma \Delta G_{\mathrm{W} \rightarrow \mathrm{Ol}}+\delta,
$$

with $\gamma=1.70,1.75$ and $\delta=2.51,4.69 \mathrm{kcal} / \mathrm{mol}$ for oneand two-bead compounds, with $R^{2}=0.97$. Given a compound's experimentally determined bulk measurement and Martini representation, $\stackrel{30}{,}$ we can thereby reconstruct the three main points of the potential of mean force, as shown in Fig. 4 a. We rationalize these findings by noting the suitability of the octanol environment as a proxy for the membrane interface. Similarly, we showed the appropriateness of octane for the bilayer midplane (Fig. 2). Indeed, both water/alcohol and water/alkane coefficients correlate with blood-brain partitioning $[39$ Therefore, the relationships in Fig. 3 stem directly from the linear correspondence between water/octane and water/octanol transfer free energies (which can be deduced from the linear relations shown in Fig. 2). From the model's perspective, the linear relations are not entirely unexpected, as Martini describes hydrophobicity by a set of equallysized Lennard-Jones particles, with varying well-depths. Interestingly, these relationships also hold at the atomistic level. At infinite dilution, the difference in partitioning of a single small molecule between water and either octane or octanol is due to a single hydrogen bond. We suspect that, at the atomistic level, the impact of this hydrogen bond on the partitioning behavior strongly informs the linearity observed, although the exact mechanism remains unclear. We further remind the reader that the relationships presented here depend strongly on the molecular weight (see the differences between one- and two-bead molecules in Fig. 3). Potentials of mean force of larger compounds ${ }^{6}$ do not follow the relationships presented in Fig. 3. Whether other relationships can be determined for these molecules will be the subject of future work.

The statistical errors displayed by the coarse-grained simulations are marginal: less than $0.1 \mathrm{kcal} / \mathrm{mol}$. However, a comparison of experimental measurements of the water/octanol partitioning free energies of several hundred small molecules against Martini predictions yielded a mean-absolute error of $0.79 \mathrm{kcal} / \mathrm{mol}^{[30}$ Given the relation between the water/octanol and water/midplane curves of Fig. 2 we deduce from it a mean absolute error on features of the potential of mean force of approximately $1.4 \mathrm{kcal} / \mathrm{mol}$. Further, the error associated with the fitted lines on Fig. 3 amounts to an overall error of roughly $1.8 \mathrm{kcal} / \mathrm{mol}$ in reconstructing the main points of the potential of mean force - at the bilayer midplane and at the interface, see circles in Fig. 4 a-by using as input only the experimental water/octanol partitioning free energy of a compound. At the atomistic level, too few potentials of mean force are available to provide errors across chemical compounds.

The linearity observed between the free-energy barrier $\Delta G_{\mathrm{W} \rightarrow \mathrm{I}}$ (equivalently $\Delta G_{\mathrm{I} \rightarrow \mathrm{M}}$ ) and the water $/$ membrane 

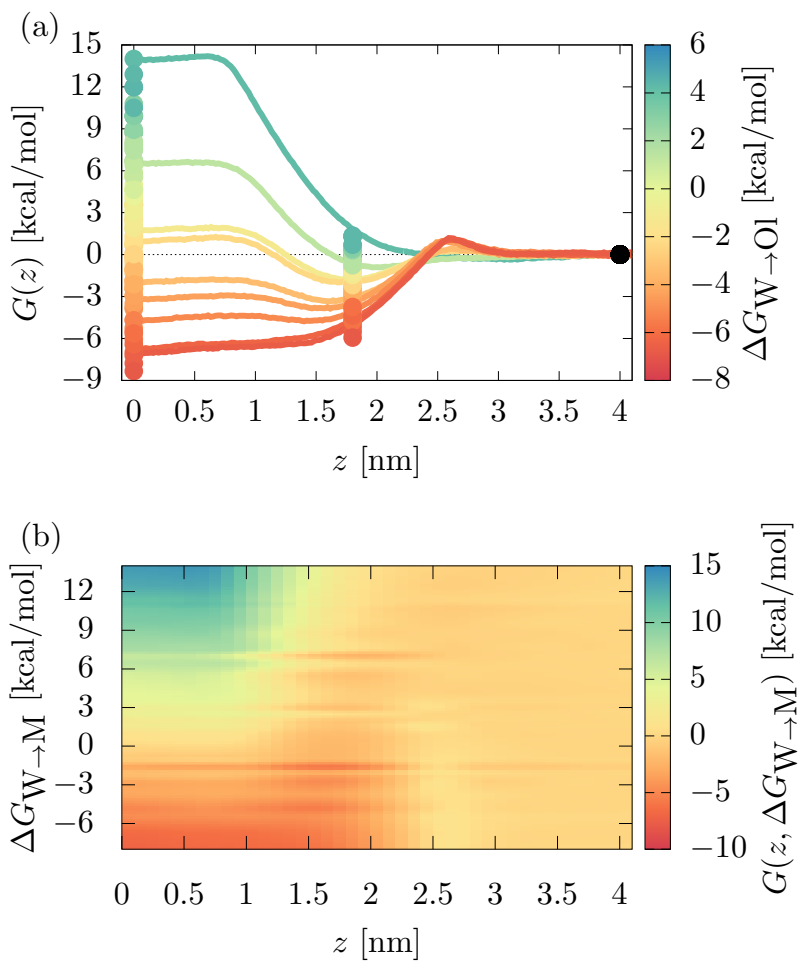

FIG. 4. (a) Representative potentials of mean force of various Martini compounds as a function of the normal distance to the bilayer midplane. The color range denotes the water/octanol partitioning of the small molecule. Large circles correspond to estimates from the thermodynamic relation extracted in this work. (b) Two dimensional map of the free energy surface $G\left(z, \Delta G_{\mathrm{W} \rightarrow \mathrm{M}}\right)$ for a small molecule, as a function of its distance from the DOPC bilayer midplane $z$ and its membrane/water partitioning free energy $\Delta G_{\mathrm{W} \rightarrow \mathrm{M}}$.

partitioning free-energy $\Delta G_{\mathrm{W} \rightarrow \mathrm{M}}$ suggests the possibility of looking for an approximately smooth two dimensional free-energy surface $G\left(z, \Delta G_{\mathrm{W} \rightarrow \mathrm{M}}\right)$ across chemical space, hence as a function of $\Delta G_{\mathrm{W} \rightarrow \mathrm{M}}$ as well as of the distance from the bilayer midplane $z$.

In the case of two-bead coarse-grained molecules, we then constructed a two dimensional map of the freeenergy surface $G\left(z, \Delta G_{\mathrm{W} \rightarrow \mathrm{M}}\right)$ starting from the set of 40 potentials of mean force that were determined by means of umbrella sampling simulations, covering a range $\Delta G_{\mathrm{W} \rightarrow \mathrm{M}} \simeq[-8,14] \mathrm{kcal} / \mathrm{mol}$. Results are shown in Fig. 4p.

The correlations shown in Fig. 3 between $\Delta G_{\mathrm{W} \rightarrow \mathrm{I}}$ and $\Delta G_{\mathrm{W} \rightarrow \mathrm{M}}$ for different compounds correspond, on this surface, to the set of points $G\left(\bar{z}=1.8 \mathrm{~nm}, \Delta G_{\mathrm{W} \rightarrow \mathrm{M}}\right)$. Apart from minor fluctuations, it is evident how the overall smoothness of the surface on the lines with constant $z$ allows us to identify the existence of an average functional relationship between $\Delta G_{\mathrm{W} \rightarrow \mathrm{M}}$ of a compound and its potential of mean force $G(z)$ for every value of $z$. As an example, a small free-energy barrier located at $z \approx 2.5 \mathrm{~nm}$ is present for all the compounds with
$\Delta G_{\mathrm{W} \rightarrow \mathrm{M}} \in[-8,0] \mathrm{kcal} / \mathrm{mol}$. Small shifts in z may result from bilayer-thickness discrepancies between atomistic and coarse-grained simulations $[30$

In this work we focused on the reconstruction of key features of the potential of mean force (i.e., the water/interface and interface/membrane transfer free energies, $\Delta G_{\mathrm{W} \rightarrow \mathrm{I}}$ and $\left.\Delta G_{\mathrm{I} \rightarrow \mathrm{M}}\right)$. The results shown in Fig. 4 $4 \mathrm{~b}$ further suggests that a knowledge of the water/membrane partitioning free energy of a compound, which can be obtained from the corresponding water/octanol one via the linear relation reported in Eq. (4), allows for a semi-quantitative reconstruction of the whole potential of mean force $G(z)$.
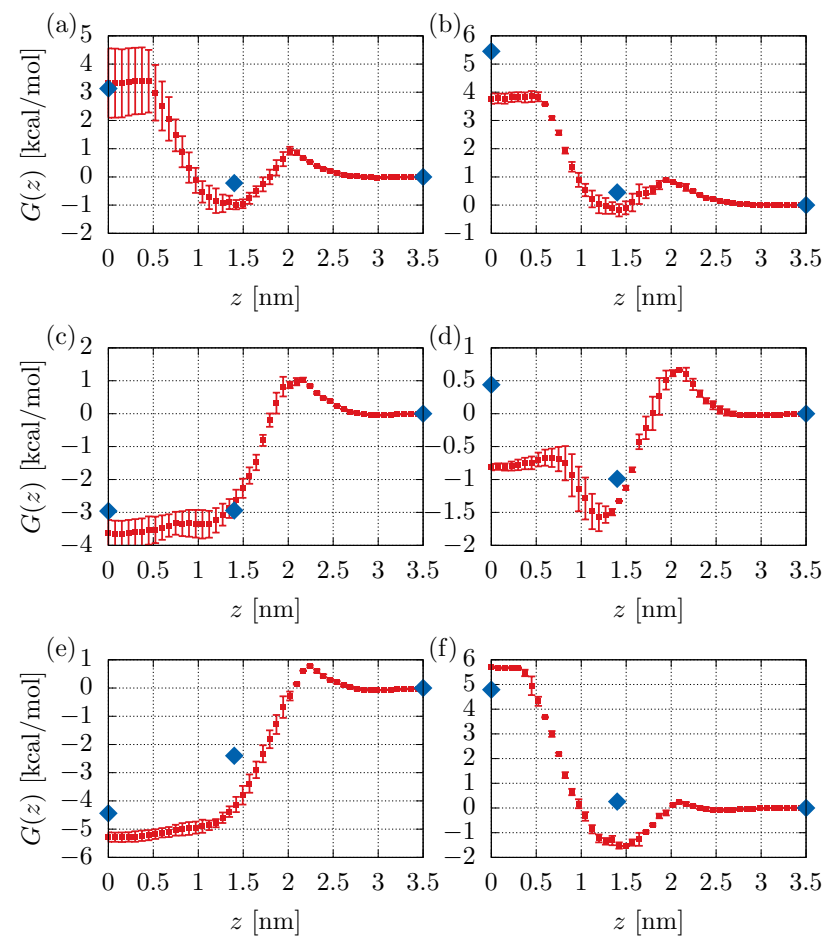

FIG. 5. Comparison between the atomistic potentials of mean force of Ref. [5] (red lines) and the predictions obtained via the linear relations presented in this work (blue squares), for the insertion of amino-acid side chains in a DOPC membrane: (a) threonine, (b) serine, (c) leucine, (d) cysteine, (e) isoleucine (ile), (f) asparagine (asn).

We now quantitatively test our results by comparing the reconstruction of the potential of mean force obtained through the linear relationships presented in Fig. 3 with the atomistic results for amino-acid side chains of Ref. 5, which map to Martini single-bead molecules. In practice, for each compound we started from the water/octanol partitioning free energy obtained by means of the ALOGPS neural network, $\frac{37}{37}$ which was then converted to a water/membrane free-energy by means of Eq. (4). We then used the linear relations of Fig. 3 to determine the water/interface and interface/midplane transfer free energies. Results are shown in Fig. 5. In 
the first four panels, corresponding to threonine, serine, leucine and cysteine, our predicted free energies are in good agreement with the atomistic results (within our 1.8 $\mathrm{kcal} / \mathrm{mol}$ error estimate). Therefore, our method based on coarse-grained simulations is capable of providing a good approximation to key features of the atomistic potential of mean force. On the other hand, by means of an integrated measure on the potential of mean force, atomistic results were shown to accurately reproduce the experimental transfer free energy between water and membrane for different compounds. $\frac{9}{9}$ The agreement between coarse-grained predictions and atomistic/experimental results has been already described in detail in previous works $18 \mid 30$ These results further suggest the important role of coarse-grained models as a reasonably predictive tool to capture the fundamental properties of biological soft matter. In panels (e) and (f) of Fig. 5 we show the case of isoleucine (ile) and asparagine (asn), two of the three statistically significant outliers observed in the linear relations presented in Fig. 3. As a consequence, the predicted free energies do not agree with the atomistic results within our error estimate. The origin of these discrepancies is very different in the two cases, and deserves to be discussed separately. The water/membrane free energy of isoleucine lies outside the interval covered by the Martini single-bead compounds, i.e., outside the range of free energies in which linearity is observed, due to the limited number of Martini beads (see Fig. 3). The introduction of more apolar beads in Martini could solve this issue, and work in this direction is in progress. In the case of asparagine and glutamine, which display similar potentials of mean force, the water/interface (and consequently interface/membrane) free-energy is underestimated if one uses the linear relation observed for single-bead compounds (see Fig. 3 and Fig. 5 , panel (f)). More interestingly, the datapoints corresponding to these molecules are in agreement with those of two-bead compounds. As both molecules are composed by a combination of a polar and an apolar group, this suggests that their representation in terms of spherical single-bead molecules is incapable of capturing their amphipathic nature, which in turn generates an underestimation of the predicted freeenergy barriers. Therefore, the natural coarse-grained representation of both compounds would be that of a two-bead molecule, as Fig. 3 suggests.

\section{B. Coarse-graining reduces the size of chemical compound space}

Alternatively to the method presented in Sec. IIIA, we identify an independent means to estimate the potential of mean force. We recently showed how the Martini model groups molecules into fewer coarse-grained representations, thereby effectively reducing the size of chemical compound space $\$ 30$ This grouping stems from the discrete set of bead types of the Martini model, which assigns the same representation to groups that are chem-

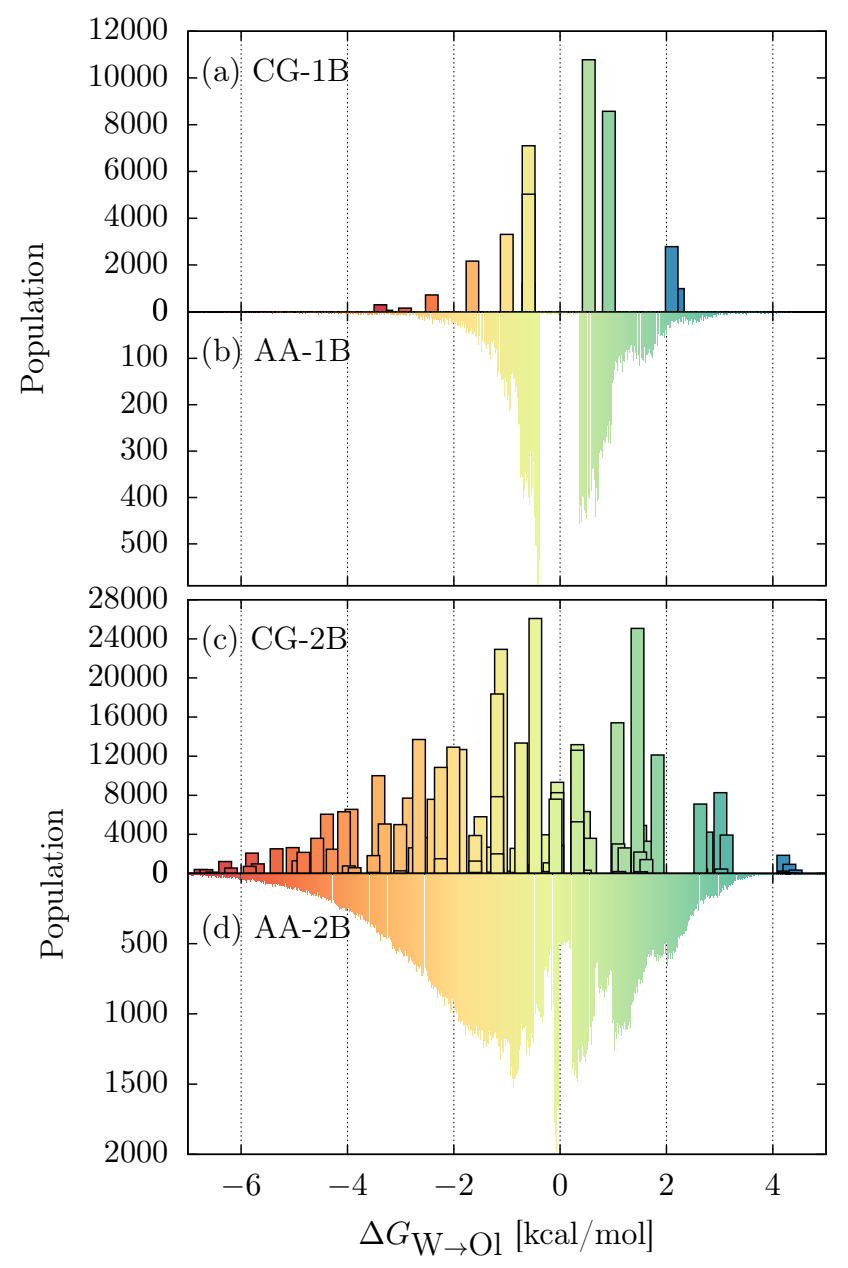

FIG. 6. Histograms of 465,387 small molecules extracted from GDB that map onto one-bead or two-bead coarse-grained representations. (a),(c) Coarse-grained and (b),(d) atomistic populations as a function of water/octanol partitioning free energy. The width of the bars in (a),(c) have no physical significance and are simply for the reader's convenience.

ically similar. To estimate this grouping, we have coarsegrained compounds from the Generated Database ${ }^{36}$ of molecules up to ten heavy atoms. In Fig. 6, we show the distributions of compounds that map to any one of the one- and two-bead coarse-grained representations considered here, as a function of the water/octanol partitioning. The atomistic distributions of Fig. 6b,d were obtained using the ALOGPS neural network. ${ }^{37}$ Despite the uneven spacing of the water/octanol partitioning free energies of the coarse-grained molecules, the atomistic distributions are roughly reproduced by the coarse-grained distributions in Fig. 6a,c, except for small artifacts in the strongly polar regime (i.e., $\Delta G_{\mathrm{W} \rightarrow \mathrm{Ol}} \gtrsim 2.0 \mathrm{kcal} / \mathrm{mol}$ ). In 
total, we identified 465,387 unique molecules, representing most synthetically-feasible small organic molecules between 30 and 160 Da. This many-to-few mapping arises solely from the limited representability of thermodynamic properties of chemical groups, rather than the coarser structural representation (i.e., atoms to beads). The removal of chemically and structurally specific information present in atomistic simulations is traditionally viewed as a necessary drawback for access to otherwise computationally prohibitive simulations. However, it is precisely this drawback that enables a single coarse-grained simulation to be representative of a large number of small molecules, as degenerate chemical groups are mapped to the same bead type. As a counterexample, fixing a Martini-like mapping in combination with a non-transferable, chemically-specific parametrization (e.g., as in most bottom-up, structure-based models) would prevent any reduction in chemical space. This work thereby introduces the ability for transferable coarse-grained models to screen large numbers of small molecules.

\section{Permeability coefficient}

Beyond the structural resolution of a large number of compounds across a membrane interface, the present results provide valuable information for other properties of interest. For instance, the permeability coefficient, $\underline{40 / 41}$ $\log P$ - a measure for passive permeation that relies on the potential of mean force-has been shown to better correlate with blood-brain barrier permeability compared to water/octanol partitioning (correlation of 0.7 and 0.9 , respectively) ${ }^{6}$ In the framework of the inhomogeneous solubility-diffusion model,, 4011 the permeability coefficient $\log P$ of a compound is defined as 7

$$
\frac{1}{P}=\int_{0}^{L} \mathrm{~d} z \frac{\exp [\beta G(z)]}{D(z)},
$$

where $D(z)$ is the position-dependent diffusion coefficient. The integration boundaries correspond to the bilayer midplane $(z=0)$ and the membrane extension $(z=L)$.

While the computational investment of atomistic simulations prevents the estimation of the potential of mean force for many compounds, $\sqrt[742]{ }$ we show that coarsegrained simulations can provide an efficient proxy. Indeed, as a first test of the accuracy of a coarse-grained potential of mean force in reproducing the permeability of a compound, we computed the $\log P$ value of mannitol in a DOPC membrane at $T=323 K$, and compared it to reference atomistic results ${ }^{[6]}$ We assumed a uniform diffusion coefficient $D(z)=D \approx 0.85 \cdot 10^{-6} \mathrm{~cm}^{2} / \mathrm{s}$, by performing a graphical extrapolation of the average value reported in Ref. 6. We obtained $\log P=-6.35$, in very close agreement with the atomistic value of -6.62. Although assuming a constant diffusion coefficient repre- sents an approximation, $D(z)$ was shown to be essentially uniform inside the membrane environment and for different compounds!

\section{CONCLUSIONS}

Determing the potential of mean force of the insertion of a small molecule in a lipid bilayer is highly informative but computationally demanding. In this work, we employed high-throughput coarse-grained molecular dynamics simulations in order to perform a screening across small molecules of small molecular weight $(\approx 30-$ $160 \mathrm{Da})$. We establish simple relationships relating the water/octanol partitioning coefficient to key features of the potential of mean force. More specifically, this study allows for a semi-quantitative estimation given a widely available experimental measurement - the water/octanol partitioning coefficient, and the coarse-grained representation in terms of Martini beads. Reference all-atom simulations found in the literature confirm these relationships for the range of molecular weights considered. The potential of mean force thereby becomes an easily accessible quantity in drug screening applications, and may be employed in various contexts, such as predicting permeabilities.

Further, any potential of mean force determined at the coarse-grained level is informative of not one, but rather a large number of small molecules. This unexpected property arises due to the coarse-grained model's limited number of bead types, which amounts to an identical representation of chemically-similar molecules. From a mathematical perspective, the present mapping from atomistic to coarse-grained molecular representations is surjective - "many-to-one." The size of chemical compound space shrinks with the properties of the coarse-grained model. We thus foresee transferable coarse-grained models to play a role in exploring chemical compound space.

We hope that high-throughput coarse-grained molecular dynamics simulations will further bring about novel insight into the thermodynamic properties of small molecules in complex environments.

\section{ACKNOWLEDGMENTS}

We thank Igor Tetko for providing a copy of the ALOGPS software, $\stackrel{37}{ }$ and Joseph F. Rudzinski and Karsten Kreis for critical reading of the manuscript. We acknowledge funding from the Emmy Noether program of the Deutsche Forschungsgemeinschaft (DFG).

\footnotetext{
${ }^{1}$ D. Lopes, S. Jakobtorweihen, C. Nunes, B. Sarmento, and S. Reis, Progress in Lipid Research 65, 24 (2016).

${ }^{2}$ W. Shinoda, Biochimica et Biophysica Acta - Biomembranes 1858, $2254(2016)$

${ }^{3}$ S. S. F. Leung, D. Sindhikara, and M. P. Jacobson, J. Chem. Inf. Model. 56, 924 (2016)
} 
${ }^{4} \mathrm{R}$. B. V. Breemen and Y. Li, Expert opinion on drug metabolism \& toxicology 1, 175 (2016)

${ }^{5}$ J. L. MacCallum, W. D. Bennett, and D. P. Tieleman, Biophys. J. 94, 3393 (2008).

${ }^{6}$ T. S. Carpenter, D. A. Kirshner, E. Y. Lau, S. E. Wong, J. P. Nilmeier, and F. C. Lightstone, Biophys. J. 107, 630 (2014)

${ }^{7}$ C. T. Lee, J. Comer, N. L. Conner Herndon, A. Pavlova, R. V. Swift, C. Tung, C. N. Rowley, R. E. Amaro, C. Chipot, Y. Wang, and J. C. Gumbart, J. Chem. Inf. Model. 56, 721 (2016).

${ }^{8}$ B. J. Bennion, N. A. Be, M. W. McNerney, V. Lao, E. M. Carlson, C. A. Valdez, M. A. Malfatti, H. A. Enright, T. H. Nguyen, F. C. Lightstone, and T. S. Carpenter, The Journal of Physical Chemistry B 121, 5228 (2017).

${ }^{9}$ S. Jakobtorweihen, A. C. Zuniga, T. Ingram, T. Gerlach, F. Keil, and I. Smirnova, J. Chem. Phys. 141, 07B622_1 (2014).

${ }^{10}$ C. Neale, W. D. Bennett, D. P. Tieleman, and R. Pomès, J. Chem. Theory Comput. 7, 4175 (2011)

${ }^{11} \mathrm{G}$. A. Voth, Coarse-graining of condensed phase and biomolecular systems (CRC press: Boca Raton, FL, 2008).

${ }^{12}$ W. G. Noid, J. Chem. Phys. 139 (2013), 10.1063/1.4818908

${ }^{13}$ X. Periole and S.-J. Marrink, Biomolecular Simulations: Methods and Protocols , 533 (2013).

${ }^{14}$ S. J. Marrink and D. P. Tieleman, Chemical Society Reviews 42, 6801 (2013).

${ }^{15}$ B. Hess, C. Kutzner, D. Van Der Spoel, and E. Lindahl, J. Chem. Theory Comput. 4, 435447 (2008).

${ }^{16}$ S. J. Marrink, A. H. de Vries, and A. E. Mark, J. Phys. Chem. B 108, 750 (2004)

${ }^{17}$ S. J. Marrink, H. J. Risselada, S. Yefimov, D. P. Tieleman, and A. H. de Vries, J. Phys. Chem. B 111, 7812 (2007).

${ }^{18}$ L. Monticelli, S. K. Kandasamy, X. Periole, R. G. Larson, D. P. Tieleman, and S.-J. Marrink, J. Chem. Theory Comput. 4, 819 (2008).

${ }^{19}$ D. H. De Jong, G. Singh, W. D. Bennett, C. Arnarez, T. A. Wassenaar, L. V. Schafer, X. Periole, D. P. Tieleman, and S. J. Marrink, J. Chem. Theory Comput. 9, 687 (2013).

${ }^{20}$ D. H. De Jong, S. Baoukina, H. I. Ingólfsson, and S. J. Marrink, Comput. Phys. Commun. 199, 1 (2016)

${ }^{21}$ M. Parrinello and A. Rahman, J. Appl. Phys. 52, 7182 (1981).

${ }^{22}$ G. Bussi, D. Donadio, and M. Parrinello, J. Chem. Phys. 126, 014101 (2007).
${ }^{23}$ T. A. Wassenaar, H. I. Ingolfsson, R. A. Bockmann, D. P. Tieleman, and S. J. Marrink, J. Chem. Theory Comput. 11, 2144 (2015).

${ }^{24}$ G. M. Torrie and J. P. Valleau, J. Comput. Phys. 23, 187 (1977).

${ }^{25}$ T. Bereau, Z.-J. Wang, and M. Deserno, J. Chem. Phys. 140, 03B615_1 (2014).

${ }^{26}$ S. Kumar, J. M. Rosenberg, D. Bouzida, R. H. Swendsen, and P. A. Kollman, J. Comput. Chem. 13, 1011 (1992).

${ }^{27}$ T. Bereau and R. H. Swendsen, J. Comput. Phys. 228, 6119 (2009).

${ }^{28}$ J. S. Hub, B. L. De Groot, and D. Van Der Spoel, J. Chem. Theory Comput. 6, 3713 (2010).

${ }^{29}$ C. Z. Mooney, R. D. Duval, and R. Duvall, Bootstrapping: A nonparametric approach to statistical inference, 94-95 (Sage, 1993).

${ }^{30}$ T. Bereau and K. Kremer, J. Chem. Theory Comput. 11, 2783 (2015)

${ }^{31}$ C. Chipot and A. Pohorille, Free energy calculations (Springer, 2007).

${ }^{32}$ A. Nikolic, S. Baud, S. Rauscher, and R. Pomès, Proteins: Structure, Function, and Bioinformatics 79, 1 (2011).

${ }^{33}$ M. R. Shirts and J. D. Chodera, J. Chem. Phys. 129, 124105 (2008).

${ }^{34}$ C. H. Bennett, J. Comput. Phys. 22, 245 (1976).

${ }^{35}$ T. Fink, H. Bruggesser, and J.-L. Reymond, Angewandte Chemie International Edition 44, 1504 (2005).

${ }^{36}$ T. Fink and J.-l. Reymond, J. Chem. Inf. Model. 47, 342 (2007).

${ }^{37}$ I. V. Tetko, V. Y. Tanchuk, and a. E. Villa, J. Chem. Inf. Comput. Sci. 41, 1407 (2001)

${ }^{38}$ I. V. Tetko and V. Y. Tanchuk, J. Chem. Inf. Comput. Sci. 42, $1136(2002)$

${ }^{39}$ A. Toulmin, J. M. Wood, and P. W. Kenny, J. Med. Chem. 51, 3720 (2008).

${ }^{40}$ J. M. Diamond and Y. Katz, J. Membrane Biol. 17, 121 (1974).

${ }^{41}$ S.-J. Marrink and H. J. Berendsen, J. Phys. Chem. 98, 4155 (1994).

${ }^{42}$ R. V. Swift and R. E. Amaro, Chemical biology \& drug design 81, 61 (2013). 